\title{
In IgA Nephropathy, Glomerulosclerosis Is Associated with Increased Urinary CD80 Excretion and Urokinase-Type Plasminogen Activator Receptor-Positive Podocyturia
}

\author{
Hernán Trimarchi ${ }^{a} \quad$ Romina Canzonieri ${ }^{b} \quad$ Amalia Schiel $^{b}$ \\ Cristian Costales-Collaguazo ${ }^{c}$ Aníbal Stern $^{b}$ Matías Paulero $^{a}$ \\ Tatiana Rengel $^{a} \quad$ José Andrews $^{a} \quad$ Alejandro Iotti $^{d}$ Mariano Forrester ${ }^{a}$ \\ Fernando Lombia ${ }^{a}$ Vanesa Pomeranz ${ }^{a}$ Romina Iriarte ${ }^{a}$ Alexis Muryan ${ }^{b}$ \\ Elsa Zottac

\begin{abstract}
a Nephrology Service, Hospital Británico de Buenos Aires, Buenos Aires, Argentina;
${ }^{b}$ Central Laboratory, Hospital Británico de Buenos Aires, Buenos Aires, Argentina;

'FIBIO Houssay, CONICET, Physiopathology, Pharmacy, and Biochemistry Faculty, Universidad de Buenos Aires, Buenos Aires, Argentina; d Pathology Service, Hospital Británico de Buenos Aires, Buenos Aires, Argentina
\end{abstract}

\section{Keywords}

IgA nephropathy · Glomerulonephritis · Glomerulosclerosis · CD80 · Urokinase-type plasminogen activator receptor $\cdot$ Podocyturia $\cdot$ Proteinuria $\cdot$ Integrins

\begin{abstract}
Background: Podocyturia may determine the evolution to podocytopenia, glomerulosclerosis, and renal failure. According to the Oxford classification of IgA nephropathy (IgAN), the S1 lesion describes glomerulosclerosis. Urokinase-type plasminogen activator receptor (UPAR) participates in podocyte attachment, while CD80 increases in glomerulosclerosis. We measured uPAR-positive urinary podocytes and urinary CD80 (uCD80) in controls and in IgAN subjects with M1E0SOTO and M1EOS1TO Oxford scores to assess a potential association between podocyturia, inflammation, and glomerulosclerosis. Methods: The groups were as follows: controls (G1), $n=20$ and IgAN group (G2), $n=39$, subdivided into M1EOSOT0 (G2A), $n=$ 21 and M1E0S1TO (G2B), $n=18$. Among the included variables, we determined uPAR-positive podocytes/gram of urinary creatinine ( $\mathrm{gUrCr}$ ) and $\mathrm{uCD} 80 \mathrm{ng} / \mathrm{gUrCr}$. Biopsies with interstitial fibrosis and tubular atrophy $<10 \%$ were included. Results: Groups were not different in age and gender; urinary protein-creatinine (UP/C) ratio, Chronic Kidney Disease-Epidemiology Collaboration (CKD-EPI) equation, UPAR-positive podocytes/gUrCr, and uCD80 were significantly increased in G2 versus G1. G2A and G2B were not different in age, gender, hyperten-
\end{abstract}


Trimarchi et al.: In IgA Nephropathy, Glomerulosclerosis Is Associated with Increased Urinary CD80 Excretion and uPAR-Positive Podocyturia

sion, and follow-up. G2B displayed significantly higher UP/C, UPAR-positive podocytes, UCD80, and lower CKD-EPI versus G2A. Strong significant correlations were encountered between $\mathrm{uCD} 80$ and podocyturia in G2A and G2B. However, when G1 was compared to G2A and G2B separately, the differences with respect to UP/C, UPAR-positive podocytes, and podocyturia were significantly stronger versus G2B than versus G2A. Conclusions: IgAN presents elevated uCD80 excretion and UPAR-positive podocyturia, while CD80 correlates with podocyturia. Glomerulosclerosis (S1) at the time of biopsy is associated with higher $\mathrm{UP} / \mathrm{C}$, lower renal function, increased uPAR-positive podocyturia, and CD80 excretion, and is independent of M1. In IgAN, uPAR may participate in podocyte detachment.

(C) 2017 The Author(s)

Published by S. Karger AG, Basel

\section{Introduction}

IgA nephropathy (IgAN) has been recognized as the most common form of primary glomerulonephritis and an important cause of chronic kidney disease and end-stage kidney failure. Clinically, IgAN can present with a wide spectrum of clinical symptoms, ranging from asymptomatic microscopic hematuria to sustained proteinuria and/or rapidly progressive glomerulonephritis. A definitive diagnosis of IgAN requires a kidney biopsy, which shows dominant or codominant glomerular deposits of IgA [1]. The histologic features of IgAN are diverse, including minimal abnormalities by light microscopy, mesangial hypercellularity, endocapillary proliferation, crescents, focal or diffuse segmental and global glomerulosclerosis, and varying degrees of tubular atrophy and interstitial fibrosis [2]. The Oxford IgAN classification is the first evidencebased validated histopathologic score that has demonstrated a potential prognostic clinical impact on IgAN $[3,4]$. Among the components of this classification, the M stands for mesangial hypercellularity and the $\mathrm{S}$ for segmental glomerulosclerosis/adhesions. The designations M0 or M1 apply to mesangial hypercellularities involving $<50$ or $>50 \%$ of glomeruli, respectively, while S0 or S1 refer to the presence of segmental sclerosis at least in one glomerulus [3]. The originally proposed multihit pathogenesis model integrates findings from studies of galactosedeficient IgA1, antiglycan response, formation and deposition of IgA1-containing immune complexes, and mechanisms of immune complex-mediated tissue injury, first in the glomerulus, originally in the mesangial area, and in the interstitium later on [5]. Once deposited in the mesangium, IgA1-containing immune complexes stimulate mesangial proliferation and local production of cytokines, such as interleukin- 6 and tumor necrosis factor- $\beta[6,7]$. These molecules promote inflammatory responses by recruiting leukocytes and promote glomerular and tubulointerstitial fibrosis. Furthermore, glomerular inflammation is enhanced by the activation of the lectin or the alternative pathways of the complement system $[1,8,9]$.

It has been demonstrated that podocytopenia is related to glomerulosclerosis and progression to chronic kidney disease [10]. Indeed, the detachment of podocytes from the basement membrane, podocytopenia, and urinary excretion of podocytes has been associated with filtration barrier dysfunction in IgAN $[11,12]$.

We decided to assess two potential molecular components of the processes involved in the fourth step of the multihit model. As the role of CD80 in the inflammation cascade of glomerulosclerosis has recently been questioned $[13,14]$, our goal was to assess CD80 urinary excretion in IgAN patients with different Oxford scores and the role urokinase-type plasminogen activator receptor (UPAR) may play in the process of irreversible podocyte detachment, which determines the progression to renal failure via glomerular podocytopenia and glomerulosclerosis. Finally, we explored any potential correlation of urinary CD80 (uCD80) excretion and uPAR-positive podocyturia with the different historical biopsy scores according to the Oxford classification. 
Table 1. Comparison of different variables between controls and IgAN patients

\begin{tabular}{lccr}
\hline Variables & Group 1 $(n=20)$ & Group 2 $(n=39)$ & $p$ value \\
\hline Age, years & $30(20-48)$ & $39(17-77)$ & 0.0242 \\
Males, \% & 50 & 49 & ns \\
CKD-EPI, mL/min & $110(86-141)$ & $87(23-161)$ & 0.0148 \\
Proteinuria/creatinuria, g/g & $0.03(0.02-0.27)$ & $0.29(0.01-14.81)$ & $<0.0001$ \\
uCD80, ng/gUrCr & $17.4(0-142.01)$ & $74.72(0-526)$ & 0.0157 \\
uPAR podocytes/creatinuria, cells/g & $3.68(0-89.83)$ & $27.58(1.97-192.31)$ & $<0.0001$ \\
\hline
\end{tabular}

Values in parentheses represent range. IgAN, IgA nephropathy; CKD-EPI, Chronic Kidney DiseaseEpidemiology Collaboration; uCD80, urinary CD80; gUrCr, gram of urinary creatinine; uPAR, urokinase-type plasminogen activator receptor; ns, nonsignificant.

Table 2. Comparison of different variables between M1E0S0T0 and M1E0S1T0 IgAN patients

\begin{tabular}{lccc}
\hline Variables & Group 2A $(n=21)$ & Group 2 B $(n=18)$ & $p$ value \\
\hline Age, years & $45(17-69)$ & $33.5(18-77)$ & 0.0569 \\
Males, $n$ & $10(48)$ & $9(50)$ & ns \\
Hypertensives, $n$ & $2(10)$ & $4(23)$ & $\mathrm{ns}$ \\
Follow-up, years & $7.2(6.7-7.8)$ & $7.5(6.9-8.1)$ & $\mathrm{ns}$ \\
CKD-EPI, mL/min & $104(47-154)$ & $67.5(23-161)$ & 0.0027 \\
Proteinuria/creatinuria, g/g & $0.16(0.01-1.11)$ & $0.92(0.04-14.81)$ & 0.034 \\
uCD80, ng/gUrCr & $45.84(0-526.10)$ & $110.68(0-361.86)$ & 0.0586 \\
uPAR podocytes/creatinuria, cells/g & $17.75(1.97-192.31)$ & $36.42(8.19-17345)$ & 0.0242 \\
\hline
\end{tabular}

Values in parentheses represent range or percentage. IgAN, IgA nephropathy; CKD-EPI, Chronic Kidney Disease-Epidemiology Collaboration; uCD80, urinary CD80; gUrCr, gram of urinary creatinine; uPAR, urokinase-type plasminogen activator receptor; ns, nonsignificant.

\section{Methods}

This is a cross-sectional, observational study which included 59 individuals. Group 1 included of 20 healthy subjects without known clinical morbidities or pharmacological treatment. Potential kidney donors and subjects with normal laboratory results and clinical history were included. Group 2 consisted of 39 IgAN patients, later subdivided into group 2A, which included 21 IgAN patients with an M1E0S0T0 Oxford score, and group 2B, which included 18 patients with an M1E0S1T0 IgAN Oxford score. In all cases, IgAN was diagnosed by a percutaneous kidney biopsy. There were 18 median glomeruli per biopsy (range: 12-23). The interstitial fibrosis and tubular atrophy (IFTA) score in both groups was $<10 \%$. Vascular lesions were similar in both groups and were found only in the hypertensive patients $(1$ patient from G2A and 3 patients from G2B). Lesions consisted of mild or moderate arteriolar hyalinosis. Patient characteristics are outlined in Table 1. The elapsed time from kidney biopsy to the collection of samples in the study (time to follow-up) was approximately 7 years for both groups (Table 2). The following variables were considered: age, gender, glomerular filtration rate estimated by the Chronic Kidney Disease-Epidemiology Collaboration (CKDEPI) equation, urinary protein-creatinine $(\mathrm{uP} / \mathrm{C})$ ratio, podocyturia counted in $1020 \times$ fields observed by fluorescent microscopy adjusted per gram of creatinuria and urinary podocyte uPAR/g of creatinuria. 
Trimarchi et al.: In IgA Nephropathy, Glomerulosclerosis Is Associated with Increased Urinary CD80 Excretion and UPAR-Positive Podocyturia

\section{Podocyturia}

We have previously described the method to study podocyturia in detail in patients with Fabry disease [15]. Briefly, a midstream freshly voided urine sample was collected on-site after a minimum of $3 \mathrm{~h}$ without voiding; $20 \mathrm{~mL}$ of urine were centrifuged at $700 \mathrm{~g}$ for $5 \mathrm{~min}$ using a cytospin. The supernatant was discarded, and the obtained sediment was stored in $100-\mu \mathrm{L}$ aliquots at room temperature and mixed with a $1.5-\mathrm{mL}$ solution made of $40 \%$ paraformaldehyde diluted in phosphate-buffered saline (PBS; pH 7.2-7.4) to reach a final $10 \%$ concentration. Nuclei of podocytes were stained with 4',6-diamidino-2-phenylindole (DAPI). Podocytes were identified by immunofluorescence using synaptopodin as the primary antibody (1:100, Abcam, Cambridge, MA, USA) and IgG anti-rabbit Alexa Fluor ${ }^{\circledR} 488$ (1:100, Abcam) as the secondary antibody. Samples were analyzed employing an epifluorescent microscope Nikon Eclipse E 200. Following our standardized technique, podocytes were counted in 10 randomly chosen $20 \times$ fields of the slides, and the average of the counted podocytes in the microscopy fields was considered as the final count for each subject. The results were corrected based on the levels of urinary creatinine found in each sample [15].

CD80

The urinary concentration of CD80 was determined employing a Human CD80 Instant

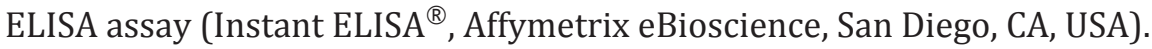

\section{UPAR Determination}

Indirect immunofluorescence colocalization by microscopy was employed for the quantification of urinary podocytes and the expression of UPAR in the same cells. In this regard, cytologic urine smears obtained from the sediment were preincubated with rabbit nonimmune serum (PBS dilution 1:100) in a humid chamber at room temperature for $1 \mathrm{~h}$. Thereafter they were incubated with the two primary antibodies: anti-synaptopodin antibody and polyclonal anti-uPAR antibody (1:200, Abcam) in a humid chamber at $4{ }^{\circ} \mathrm{C}$ overnight. Three 5 -min rinses with PBS were made, and the samples were incubated with the secondary antibodies: IgG anti-rabbit ALEXA Fluor $488^{\circledR}$ (1:100, Abcam) for synaptopodin and IgG anti-mouse uPAR Alexa Fluor ${ }^{\circledR} 568$ (1:100) for uPAR in a humid chamber for $2 \mathrm{~h}$ at room temperature. Three 5 -min rinses each were undertaken and were stained with DAPI. Samples were analyzed employing an epifluorescent microscope Nikon Eclipse E 200. The synaptopodin-uPARcostained podocytes were counted in 10 randomly chosen $20 \times$ fields of the slides as described above.

\section{Other Variables}

The values of serum creatinine and proteinuria were the ones obtained the same week in which the urine was collected for podocyte count. Creatinuria was measured from the same specimen used for the podocyte search, as follows: the value of urinary creatinine obtained from the urine was calculated for the initial urinary volume of $20 \mathrm{~mL}$ employed for the counting of podocytes. For the calculation of uP/C ratios, both variables were measured from the specimen employed for podocyte collection as well.

\section{Treatment}

After biopsy had been performed, all patients were initiated on a dual blockade of the renin-angiotensin system with daily doses of enalapril (2.5-10 $\mathrm{mg} /$ day) and valsartan (80-320 mg/day) that varied depending on patient tolerance and the degree of proteinuria. This schedule was maintained at similar doses in both groups up to the present time but changed among certain individuals, mainly the hypertensives. The aim of the dual blockade was to reach a proteinuria level $<0.5 \mathrm{~g} /$ day, if clinically tolerated. All patients with proteinuria 

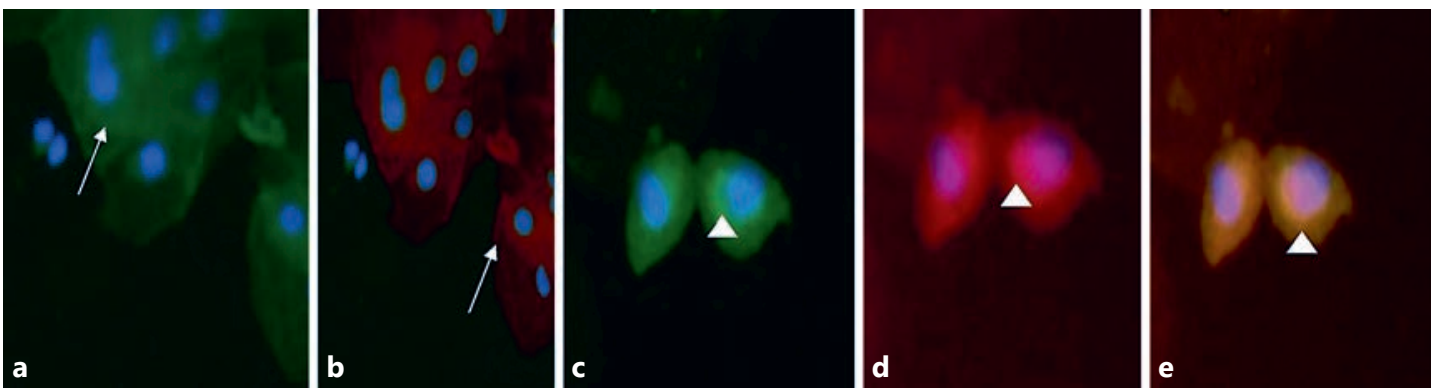

Fig. 1. The expression of uPAR in urinary podocytes of patients with IgAN. a Synaptopodin-negative cells (white arrow). b uPAR-negative cells (white arrow). c Synaptopodin-positive cells (white arrowhead). d UPAR-positive cells (white arrowhead). e Merged cells indicating the colocalization between synaptopodin and $\mathrm{UPAR}$ in IgAN podocytes. $\mathbf{a}, \mathbf{b} \times 20$. $\mathbf{c}-\mathbf{e} \times 40$.

$>1 \mathrm{~g} /$ day (group 2A $n=0$; group 2B $n=9$ ) received meprednisone for 3 months, which was tapered thereafter. No patients were on immunosuppressants at the time of the study.

\section{Ethics}

The present protocol was approved by the Institutional Review Board of the Hospital Británico de Buenos Aires, Buenos Aires, Argentina. Informed consent was obtained from each study participant.

\section{Statistical Analysis}

Results are expressed as median and range. Variables were analyzed using the Wilcoxon Mann-Whitney test. Correlations between variables were obtained with the Spearman correlation coefficient. Results were considered significant when $p<0.05$. The statistical program employed was InfoStat 2016, Córdoba, Argentina.

\section{Results}

The groups were not different with respect to age and gender. However, uP/C, uPARpositive podocytes/gram of urinary creatinine (gUrCr) uCD80, and podocyturia were significantly increased in group G2 versus group G1, while controls displayed a significantly higher renal function (Table 1; Fig. 1). All IgAN patients were on renin-angiotensin dual blockade: enalapril $2.5-10 \mathrm{mg} /$ day and valsartan $80-320 \mathrm{mg} /$ day. The doses of both drugs varied for each patient depending on tolerance to the hemodynamic status. G2A and G2B were not different as to age (45 years, range: $18-69$ vs. 33.50 years, range: 18-77), gender (males 10 vs. 9), hypertensives ( 2 vs. 4 ), and time to follow-up (7.2 years, range: 6.7-7.8 vs. 7.5 years, range: 6.9-8.1) (Table 2). G2B displayed significantly higher uP/C ratios, uPAR-positive podocyturia, uCD80 excretion, and lower renal function assessed by CKD-EPI with respect to G2A, respectively (Table 2). However, when G1 was compared to G2A and G2B separately, the differences with respect to $\mathrm{UP} / \mathrm{C}$ ratios and uPAR-positive podocytes/gUrCr were more significant against G2B, while G2A displayed an older population and a nonsignificantly higher uCD80 excretion. Finally, G2B subjects presented significantly worse renal function (Tables 3,4$)$. A strong and significant correlation was encountered between uCD80 excretion and podocyturia in G2A ( $\rho=0.73, p=0.0002)$ and in $\mathrm{G} 2 \mathrm{~B}(\rho=0.65, p=0.0036)$. A comparison of available variables at the time of biopsy and at the present time of group $2 \mathrm{~A}$ and $2 \mathrm{~B}$ subjects is depicted in Table 5. 
Trimarchi et al.: In IgA Nephropathy, Glomerulosclerosis Is Associated with Increased Urinary CD80 Excretion and uPAR-Positive Podocyturia

Table 3. Comparison of different variables between controls and M1E0S0T0 IgAN patients

\begin{tabular}{lccc}
\hline Variables & Group 1 $(n=20)$ & Group 2 A $(n=21)$ & $p$ value \\
\hline Age, years & $30(20-48)$ & $45(17-69)$ & 0.0031 \\
Males, \% & 50 & 61 & ns \\
CKD-EPI, mL/min & $110(86-141)$ & $104(47-154)$ & 0.4163 \\
Proteinuria/creatinuria, g/g & $0.03(0.02-0.27)$ & $0.16(0.01-1.11)$ & 0.0023 \\
uCD80, ng/gUrCr & $17.4(0-142.01)$ & $45.84(0-526.10)$ & 0.1161 \\
uPAR podocytes/creatinuria, cells/g & $3.68(0-89.83)$ & $17.75(1.97-192.31)$ & 0.0064 \\
\hline
\end{tabular}

Values in parentheses represent range. IgAN, IgA nephropathy; CKD-EPI, Chronic Kidney DiseaseEpidemiology Collaboration; uCD80, urinary CD80; gUrCr, gram of urinary creatinine; uPAR, urokinase-type plasminogen activator receptor; ns, nonsignificant.

Table 4. Comparison of different variables between controls and M1E0S1T0 IgAN patients

\begin{tabular}{lccc}
\hline Variables & Group 1 $(n=20)$ & Group 2B $(n=18)$ & $p$ value \\
\hline Age, years & $30(20-48)$ & $33.5(18-77)$ & 0.3875 \\
Males, \% & 50 & 33 & ns \\
CKD-EPI, mL/min & $110.0(86-141)$ & $67.5(23-161)$ & 0.0003 \\
Proteinuria/creatinuria, g/g & $0.03(0.02-0.27)$ & $0.92(0.04-14.81)$ & $<0.0001$ \\
uCD80, ng/gUrCr & $17.4(0-142.01)$ & $110.68(0-361.86)$ & 0.0063 \\
uPAR podocytes/creatinuria, cells/g & $3.68(0-89.83)$ & $36.42(8.19-17345)$ & 0.0003 \\
\hline
\end{tabular}

Values in parentheses represent range. IgAN, IgA nephropathy; CKD-EPI, Chronic Kidney DiseaseEpidemiology Collaboration; uCD80, urinary CD80; gUrCr, gram of urinary creatinine; uPAR, urokinase-type plasminogen activator receptor; ns, nonsignificant.

Table 5. Available baseline and recent variable changes in groups $2 \mathrm{~A}$ and $2 \mathrm{~B}$

\begin{tabular}{|c|c|c|c|c|c|c|}
\hline & \multicolumn{3}{|l|}{ Group 2A } & \multicolumn{3}{|l|}{ Group 2B } \\
\hline & $\begin{array}{l}\text { baseline } \\
(n=21)\end{array}$ & $\begin{array}{l}\text { recent } \\
(n=21)\end{array}$ & $\begin{array}{l}p \\
\text { value }\end{array}$ & $\begin{array}{l}\text { baseline } \\
(n=18)\end{array}$ & $\begin{array}{l}\text { recent } \\
(n=18)\end{array}$ & $\begin{array}{l}p \\
\text { value }\end{array}$ \\
\hline Hypertensives, $n$ & 2 & 2 & ns & 4 & 4 & ns \\
\hline $\begin{array}{l}\text { CKD-EPI, mL/min } \\
\text { Proteinuria/ }\end{array}$ & $112(53-123)$ & $104(47-154)$ & 0.01 & $79.94(75-113)$ & $67.5(23-161)$ & 0.001 \\
\hline creatinuria, g/g & $0.43(0.17-0.77)$ & $0.16(0.01-1.11)$ & 0.03 & $1.57(0.8-2.1)$ & $0.92(0.04-14.81)$ & $<0.0001$ \\
\hline
\end{tabular}

Values in parentheses represent range. Baseline: data obtained at the time of kidney biopsy 7.5 years earlier (6.9-8.1). CKD-EPI, Chronic Kidney Disease-Epidemiology Collaboration; ns, nonsignificant.

\section{Discussion}

Our results show that in IgAN, podocyturia and uCD80 excretion are elevated when compared to controls (Table 1). Moreover, it appears that in the process of podocyte detachment, uPAR is directly involved, due to its presence in lost podocytes (Fig. 1). With respect to $\mathrm{CD} 80$, its presence is increased and correlates with uPAR-positive podocyturia, but this could be interpreted either as an epiphenomenon or as an actively involved molecule in 
Trimarchi et al.: In IgA Nephropathy, Glomerulosclerosis Is Associated with Increased Urinary CD80 Excretion and uPAR-Positive Podocyturia

podocyturia. This speculation is based on the fact that these statements are the result of a mere measurement of CD80 in the urine. In this regard, we did not demonstrate its source of origin. However, uPAR-positive podocyturia and uCD80 excretion are both also significantly elevated in G2A and G2B despite the dual blockade. We believe these interesting findings raise an important issue: although the therapy targets the renin-angiotensin system (the only 1A evidence-based therapy indication for IgAN according to the K-DIGO guidelines) [16,17], this therapeutic approach is clinically insufficient, as podocyturia still persists and renal function deteriorates (Table 5). Our results may explain the reason why IgAN is a glomerulonephritis with a relentless profile of progression to renal insufficiency and end-stage renal disease in a high proportion of its population [18]. It has been published that $20-50 \%$ of IgAN patients require renal replacement therapy 20 years after diagnosis [18]. Interestingly our findings result from patients with a supposedly low Oxford score. Finally, kidney biopsies with the $S$ component of the Oxford classification and IFTA $<10 \%$ were associated with higher protein excretion, lower renal function, increased UPAR-positive podocyturia, and CD80 excretion. The S score is associated with a poor outcome in some cohorts of IgA patients, albeit not in others $[2,19]$.

As IgAN is a frequent cause of end-stage renal disease, this situation compels nephrologists to find early indicators of progression to avoid or delay renal replacement therapy. A milestone in the history of IgAN has been the incorporation of the Oxford classification into clinical practice. The Oxford classification is a clinically validated histopathologic tool that has the advantage of including histologic components that relate a determined lesion to a clinically validated prognostic factor and to therapeutic implications, and these correlations also portend clinical impact $[4,20]$. According to IgAN physiopathology, mesangial proliferation, a frequent biopsy finding, is supposed to antedate all the other histologic components of the classification. When IgA-IgG immune complexes are deposited in the mesangium, matrix expansion and mesangial hypercellularity take place. In this fourth hit of the disease, inflammatory components are synthesized and podocyte loss is supposed to commence. In agreement with these reports, we have found that in G2A biopsies, podocyte loss is elevated, and that CD80 excretion correlates with podocyturia.

On the other hand, sclerosis is a more advanced lesion that implies major podocyte loss and subsequent scarring due to glomerular basement membrane denudation and inflammatory processes. Glomerulosclerosis presents clinical correlators such as higher proteinuria, lower renal function, and worse outcomes. Our first goal was to assess the degree of spontaneous podocyte loss in patients with IgAN whose biopsies showed mild histologic compromise (G2A) and compared it to those with more advanced derangement (G2B) (Tables 1-4) and any potential mechanism of podocyte detachment. As expected, the $S$ component was associated with higher podocyturia. In addition, in IgAN podocytes appear to detach via UPAR activation. This finding has important implications, as any intervention that could impede uPAR coupling with $\alpha \mathrm{V} \beta 3$ or $\alpha 3 \beta$ integrins and podocyte contraction would result in lower podocyte loss and glomerular sclerosis $[21,22]$. In this regard, amiloride is a drug that inhibits certain integrin subunits and interferes with uPAR synthesis [23].

The M1 score is generally present in all IgAN biopsies and is related to a worse prognosis in the long term, although it is an early event and an initial histologic pattern of disease [4]. Despite our lack of IgAN subjects with M0 to compare it with, according to Wiggings [10] this proliferative event is accompanied by a silent and irreversible podocyte loss. Moreover, CD80 is increased and correlates with podocyturia, suggesting either an inflammatory process mediated by a coligand signal between CD4+ lymphocytes and antigen-presenting cells or else an upregulation of CD80 in affected podocytes. In any case, this increase in CD80 urinary excretion may indicate another potential target for therapeutic intervention. 
In addition to the above-mentioned causes of worse renal function in S1 versus S0 patients, hypertension could also be an additional factor. Although no significant difference in the number of hypertensive subjects was found in both groups (Table 2), the arteriolar hyalinosis reported many years before could have played a role in the progression of renal disease. Besides, a higher number of subjects should be included to assess this hypothesis.

Finally, we measured the levels of uCD80 in this cohort and assessed any correlation with sclerosis. Noteworthy, we excluded patients whose biopsies displayed IFTA $>10 \%$, in order to avoid any other potential sclerotic source of CD80 synthesis. Not only IgAN subjects displayed higher uCD80 excretion when compared to controls, but the $S$ component was also associated with higher CD80 levels. As we did not perform biopsy immunohistochemical staining, we cannot make any statements regarding the source of CD80. Recently, the role of CD80 in the pathogenesis of some glomerular diseases such as minimal change disease and primary focal and segmental gomerulosclerosis has been seriously questioned [13]. Wu et al. [24] have encountered higher concentrations of CD80+ cells in IgAN human biopsies. As recently commented by Novelli et al. [25], despite the reported evidence that the expression of CD80 could be induced on podocytes under stress conditions such as lupus nephritis, membranous and diabetic nephropathies, and minimal change disease and primary focal and segmental gomerulosclerosis, the authors have demonstrated that at least in the case of minimal change disease and primary focal and segmental gomerulosclerosis, this finding was based on unreliable immunohistochemical assays for CD80. Finally, we also reported increased levels of uCD80 in Fabry patients, and we propose podocytes as a potential source due to our in vitro findings and CD80+ podocyte immunohistochemical staining in a kidney biopsy [26].

Our paper presents several limitations. While the search for urinary podocytes is noninvasive, it is also time-consuming and may be erratic [15, 27, 28]. Moreover, it needs to be validated with studies adjusted for kidney function, age, and probably also for the type of glomerulopathy. We believe that in the case of IgAN, it has to be assessed at each of the different Oxford scores. In our study, only one marker was employed to identify podocytes (synaptopodin), but certainly the complementation of other podocyte markers such as podocalyxin, podocin, or Wilms tumor 1 protein might have potentially identified podocytes at other cell cycle stages [29]. All these considerations underscore the need to standardize the study of podocyturia. Moreover, uPAR mRNA must be obtained from these podocytes to confirm our qualitative findings. We believe that podocyturia is a key marker to study the pathophysiology of glomerular diseases and a useful tool to assess therapeutic interventions. Another pending answer is whether immunosuppression could modify the degree of podocyturia or decrease the urinary levels of CD80 and/or UPAR. This would be an interesting topic for future research.

Finally, against the findings of this study, it could be argued that the time gap between the biopsy date and the determination of podocyturia was approximately 7 years and that the sclerotic findings could not have an association with podocyturia. However, it must be borne in mind that both glomerulosclerosis (S1) and podocyturia are irreversible phenomena [2, 21]. In this regard, it could also be speculated that a second kidney biopsy would still present the S1 score. Finally, as no evidence-based recommendation exists to rebiopsy patients with IgAN unless there is a rapid change in the clinical course exists, we assume that the IFTA score $<10 \%$ may not have changed significantly, albeit the chance of a change in the MEST score could also be a possibility. However, we have demonstrated that an initial S1 score is accompanied by a higher urinary podocyte excretion rate, proteinuria, uCD80 excretion, and lower renal function over time (Table 1).

In conclusion, in IgAN, CD80 correlates with podocyturia, while uPAR-positive urinary podocytes and uCD80 excretion are increased in the M1E0S0T0 and M1E0S1T0 Oxford 
Trimarchi et al.: In IgA Nephropathy, Glomerulosclerosis Is Associated with Increased Urinary CD80 Excretion and uPAR-Positive Podocyturia

scores. The $\mathrm{S} 1$ lesion is associated with higher proteinuria, lower renal function, increased uPAR-positive podocyturia, and uCD80 excretion. In IgAN, uPAR may participate in podocyte detachment at initial stages of the disease and eventually in disease progression.

\section{Acknowledgements}

We would like to acknowledge Ms. Laura Ares and Ms. Marina Fernandez for their professional assistance.

\section{Disclosure Statement}

The authors have no conflicts of interest to disclose.

\section{References}

1

$\nabla_{2}$ IS: Pathology of IgA nephropathy. Nat Rev Nephrol 2014:10:445-454

Hill GS, Karoui KE, Karras A, et al: Focal segmental glomerulosclerosis plays a major role in the progression of IgA nephropathy. Immunohistochemical studies. Kidney Int 2011;79:635-642.

-3 Working Group of the International IgA Nephropathy Network and the Renal Pathology Society, Roberts IS, Cook HT, Troyanov S, et al: The Oxford classification of IgA nephropathy: pathology definitions, correlations, and reproducibility. Kidney Int 2009;76:546-556.

-4 Working Group of the International IgA Nephropathy Network and the Renal Pathology Society, Cattran DC, Coppo R, Cook HT, et al: The Oxford classification of IgA nephropathy: rationale, clinicopathological correlations, and classification. Kidney Int 2009;76:534-545.

-5 Suzuki H, Kiryluk K, Novak J, et al: The pathophysiology of IgA nephropathy. J Am Soc Nephrol 2011;22:17951803.

-6 Novak J, Julian BA, Mestecky J, et al: Glycosylation of IgA1 and pathogenesis of IgA nephropathy. Semin Immunopathol 2012;34:365-382.

7 Lai KN: Pathogenesis of IgA nephropathy. Nat Rev Nephrol 2012;8:275-283.

-8 Jennette JC: The immunohistology of IgA nephropathy. Am J Kidney Dis 1988;12:348-352.

9 Haas M: IgA nephropathy and Henoch-Schonlein purpura nephritis; in Jennette JC, D’Agati VD, Olson JL, et al (eds): Heptinstall's Pathology of the Kidney, ed 7. Philadelphia, Lippincott Williams \& Wilkins, 2015, pp 475-524.

10 Wiggins RC: The spectrum of podocytopathies: a unifying view of glomerular diseases. Kidney Int 2007;71: 1205-1214.

11 Lemley KV, Lafayette RA, Safai M, et al: Podocytopenia and disease severity in IgA nephropathy. Kidney Int 2002;61:1475-1485.

-12 Hara M, Yanagihara T, Kihara I: Cumulative excretion of urinary podocytes reflects disease progression in IgA nephropathy and Schönlein-Henoch purpura nephritis. Clin J Am Soc Nephrol 2007;2:231-238.

13 Kronbichler A, Leierer J, Oh J, Meijers B Il, Shin J: Immunologic changes implicated in the pathogenesis of focal segmental glomerulosclerosis. Biomed Res Int 2016;2016:2150451.

14 Salant DJ: Podocyte expression of B7-1/CD80: is it a reliable biomarker for the treatment of proteinuric kidney diseases with Abatacept? J Am Soc Nephrol 2016;27:963-965.

-15 Trimarchi H, Canzonieri R, Schiel A, et al: Podocyturia in Fabry adult untreated and treated patients. A controlled study. J Nephrol 2016;6:791-797.

-16 Cattran DC, Feehally J, Cook HT, et al: Kidney disease: improving global outcomes (KDIGO) glomerulonephritis work group. KDIGO clinical practice guideline for glomerulonephritis. Kidney Int Suppl 2012;2:139-274.

-17 Rauen T, Eitner F, Fitzner C, et al: Intensive supportive care plus immunosuppression in IgA nephropathy. N Engl J Med 2015;373:2225-2236.

-18 Schena FP: A retrospective analysis of the natural history of primary IgA nephropathy worldwide. Am J Med 1990;89:209-215.

19 Bellur SS, Lepeytre F, Vorobyeva O, et al: Evidence from the Oxford Classification cohort supports the clinical value of subclassification of focal segmental glomerulosclerosis in IgA nephropathy. Kidney Int 2017;91:235241.

20 Working Group of the International IgA Nephropathy Network and the Renal Pathology Society, Coppo R, Troyanov S, Camilla R, et al: The Oxford IgA nephropathy clinicopathological classification is valid for children as well as adults. Kidney Int 2010;77:921-927. 
Trimarchi et al.: In IgA Nephropathy, Glomerulosclerosis Is Associated with Increased Urinary CD80 Excretion and uPAR-Positive Podocyturia

21

$-22$

Trimarchi H: Podocyturia. What is in a name? J Transl Int Med 2015;3:51-56.

Kobayashi N, Ueno T, Ohashi K, et al: Podocyte injury-driven intracapillary plasminogen activator inhibitor type 1 accelerates podocyte loss via uPAR-mediated $\beta_{1}$-integrin endocytosis. Am J Physiol Renal Physiol 2015;308:F614-F626.

23 Zhang B, Xie S, Shi W, Yang Y: Amiloride off-target effect inhibits podocyte urokinase receptor expression and reduces proteinuria. Nephrol Dial Transplant 2012;27:1746-1755.

$24 \mathrm{Wu} \mathrm{Q}$, Jinde K, Endoh M: Clinical significance of costimulatory molecules CD80/CD86 expression in IgA nephropathy. Kidney Int 2004;65:888-896.

-25 Novelli R, Gagliardini E, Ruggiero B, et al: Any value of podocyte B7-1 as a biomarker in MCD and FSGS? Am J Physiol Renal Physiol 2016;310:F335-F341.

-26 Trimarchi H, Canzonieri R, Schiel A, et al: Increased urinary CD-80 excretion and podocyturia in Fabry disease. J Transl Med 2016;14:289.

27 Trimarchi H, Canzonieri R, Muryan A, et al: Copious podocyturia without proteinuria and with normal renal function in a young adult with Fabry disease. Case Rep Nephrol 2015;2015:257628.

28 Nakamura T, Ushiyama C, Suzuki S, et al: Urinary excretion of podocytes in patients with diabetic nephropathy. Nephrol Dial Transplant 2000;15:1379-1383.

29 Maestroni S, Maestroni A, Dell'Antonio G, et al: Viable podocyturia in healthy individuals: implications for podocytopathies. Am J Kidney Dis 2014;64:1003-1005. 